\title{
Radiomics-based machine learning methods for isocitrate dehydrogenase genotype prediction of diffuse gliomas
}

\author{
Shuang $\mathrm{Wu}^{1,2} \cdot$ Jin Meng ${ }^{1,2} \cdot \mathrm{Qi} \mathrm{Yu^{1,2 }} \cdot$ Ping $\mathrm{Li}^{3} \cdot$ Shen $\mathrm{Fu}^{1,2,3,4,5}$
}

Received: 16 August 2018 / Accepted: 1 November 2018 / Published online: 4 February 2019

(c) The Author(s) 2019

\begin{abstract}
Purpose Reliable and accurate predictive models are necessary to drive the success of radiomics. Our aim was to identify the optimal radiomics-based machine learning method for isocitrate dehydrogenase (IDH) genotype prediction in diffuse gliomas. Methods Eight classical machine learning methods were evaluated in terms of their stability and performance for pre-operative IDH genotype prediction. A total of 126 patients were enrolled for analysis. Overall, 704 radiomic features extracted from the pre-operative MRI images were analyzed. The patients were randomly assigned to either the training set or the validation set at a ratio of 2:1. Feature selection and classification model training were done using the training set, whereas the predictive performance and stability of the model were independently assessed using the validation set.

Results Random Forest (RF) showed high predictive performance (accuracy $0.885 \pm 0.041$, AUC $0.931 \pm 0.036$ ), whereas neural network (NN) (accuracy $0.829 \pm 0.064$, AUC $0.878 \pm 0.052$ ) and flexible discriminant analysis (FDA) (accuracy $0.851 \pm 0.049$, AUC $0.875 \pm 0.057$ ) displayed low predictive performance. With regard to stability, RF also showed high robustness against data perturbation (relative standard deviations, RSD 3.87\%).

Conclusions RF is a promising machine learning method in predicting IDH genotype. Development of an accurate and reliable model can assist in the initial diagnostic evaluation and treatment planning for diffuse glioma patients.
\end{abstract}

Keywords Radiomics $\cdot$ Isocitrate dehydrogenase $\cdot$ Diffuse glioma $\cdot$ Magnetic resonance imaging $\cdot$ Machine learning

\section{Introduction}

Gliomas account for approximately $70 \%$ of malignant central nervous system (CNS) tumors (Ostrom et al. 2017). According to the 2016 World Health Organization (WHO) classification of CNS tumors, adult diffuse gliomas consist of astrocytic tumors, oligodendrogliomas, and glioblastomas (WHO grade II-IV) (Louis et al. 2016). Genomic characterization has demonstrated that identifications of mutations in isocitrate dehydrogenase (IDH) genes were associated with improved prognosis in patients with glioma (Cancer Genome Atlas Research et al. 2015; Hartmann et al. 2010; Parsons et al. 2008; Yan et al. 2009). The median overall survival of patients with IDH-mutated glioblastoma was 31 months compared to 15 months for those without the mutation (Yan et al. 2009). Patients diagnosed with IDH wild-type grade II-III glioma, which was molecularly and clinically similar to glioblastoma, had worse overall survival than those with IDH-mutated glioma of same grade (Cancer Genome Atlas Research et al. 2015). It thus seems that the identification of IDH genotype is important in the management of gliomas.

5 Department of Radiation Oncology, Shanghai Concord Cancer Hospital, Shanghai 200020, China 
At present, the most commonly used method to assess IDH mutation status is molecular assay following biopsy or surgical resection. Although molecular assay can be informative, there are many factors that can limit its clinical use in evaluating treatment response and monitoring cancer progression (Rios Velazquez et al. 2017). These limiting factors include the lack of regular biopsies or surgical resections at the end of each treatment course, difficulty in evaluating the intra-tumor heterogeneity, inconvenient access of tumor samples, and failure to identify molecular genotype due to the poor quality of tumor tissues. In contrast to molecular assays, magnetic resonance imaging (MRI) is routinely used in the initial diagnosis and treatment response assessment of gliomas. Taking full advantage of the abundant information in these easily accessible images may provide an opportunity to overcome the limitations related to molecular assay.

MRI features have been used to predict the clinical outcomes and molecular subtypes including IDH genotype in glioma (Carrillo et al. 2012; Lee et al. 2015; Park et al. 2017). However, only a few imaging features have been used. Moreover, the identification of qualitative features is often inconsistent between observers. "Radiomics", an emerging and promising field, hypothesizes that the advanced analysis of medical images can capture hundreds of additional features which are not currently used and may be valuable in personalized medicine (Lambin et al. 2012). Several studies have investigated the potential of these high-dimensional and minable radiomic features to noninvasively facilitate tumor detection, subtype classification, therapeutic response assessment, and prognosis prediction in multiple cancers (Aerts et al. 2014; Fehr et al. 2015; Huang et al. 2016a, b; Li et al. 2016; Nie et al. 2016; Rios Velazquez et al. 2017; Zhang et al. 2017). For gliomas, radiomic features have also been applied to predict patient survival and molecular subtypes via machine learning methods (Macyszyn et al. 2016; Rathore et al. 2016; Yu et al. 2017; Zhang et al. 2017a, b, 2018). However, the effectiveness of different radiomicsbased machine learning approaches in IDH genotype prediction for patients with diffuse glioma is yet to be assessed.

Using radiomic features provided in the TCGA/TCIA repositories (Bakas et al. 2017a, b), we evaluated and compared eight classical machine learning methods in terms of their stability and performance for noninvasive and pre-operative IDH genotype prediction. A total of 126 patients with diffuse glioma were enrolled for analyses. Feature selection and classification model training were performed using the training set. The predictive performance of the model was independently tested in the validation set. Our aim was to identify the optimal and reliable IDH genotype prediction methods with the full use of MRI images.

\section{Materials and methods}

\section{Data collection and patient cohort}

All the patients included in our study were de-identified by the Cancer Genome Atlas; hence, no Health Insurance Portability and Accountability Act or institutional review board approval was required. Radiomic features and additional information of the TCGA-GBM and TCGA-LGG collections consisting of 102 and 65 (Bakas et al. 2017a, b) patients, respectively, were obtained from the TCIA database (Clark et al. 2013) and the related articles (Bakas et al. 2017c). IDH genotype and clinical information were acquired from a molecular profiling study of diffuse gliomas (Ceccarelli et al. 2016). We correlated the subtypes and clinical information with the imaging features using the unique TCGA identifiers. Patients who met the following criteria were included in this study: (1) available radiomic features from the enhancing part of the tumor core (ET), the non-enhancing part of the tumor core (NET), and the peritumoral edema region (ED) of pre-operative MRI images, (2) known IDH genotype, and (3) age and gender information available at diagnosis (Fig. 1). Our final cohort consisted of 126 patients with grade II-III $(n=43)$ and grade IV $(n=83)$ glioma (Table 1$)$.

\section{Radiomic features}

The details of image processing are described in a previous study (Bakas et al. 2017c). The pre-operative MRI modalities, which included pre-contrast T1-weighted (T1), postcontrast T1-weighted (T1-Gd), T2-weighted (T2), and T2 fluid-attenuated inversion recovery (T2-FLAIR), were used for image analysis. Three sub-regions (ET, NET, ED) were segmented by an automated method and manually revised by an experienced neuroradiologist. The intensity features, volumetric features, histogram-based features, textural parameters, spatial features, and glioma diffusion properties based on glioma growth models were calculated. The textural parameters were derived from the gray-level co-occurrence matrix (GLCM), gray-level run-length matrix (GLRLM), gray-level size zone matrix (GLSZM), and neighborhood gray-tone difference matrix (NGTDM). Finally, 704 radiomic features were extracted.

\section{Feature selection and classification methods}

Eight classical machine learning methods were investigated and compared: Random Forest (RF), adaptive boosting (Adaboost), Naive Bayes (NB), flexible discriminant analysis (FDA), k-nearest neighbors (kNN), support vector machines with radial basis function kernel (r-SVM), support vector machines with linear kernel (1-SVM), and neural 
Fig. 1 Workflow of classification method training and validation

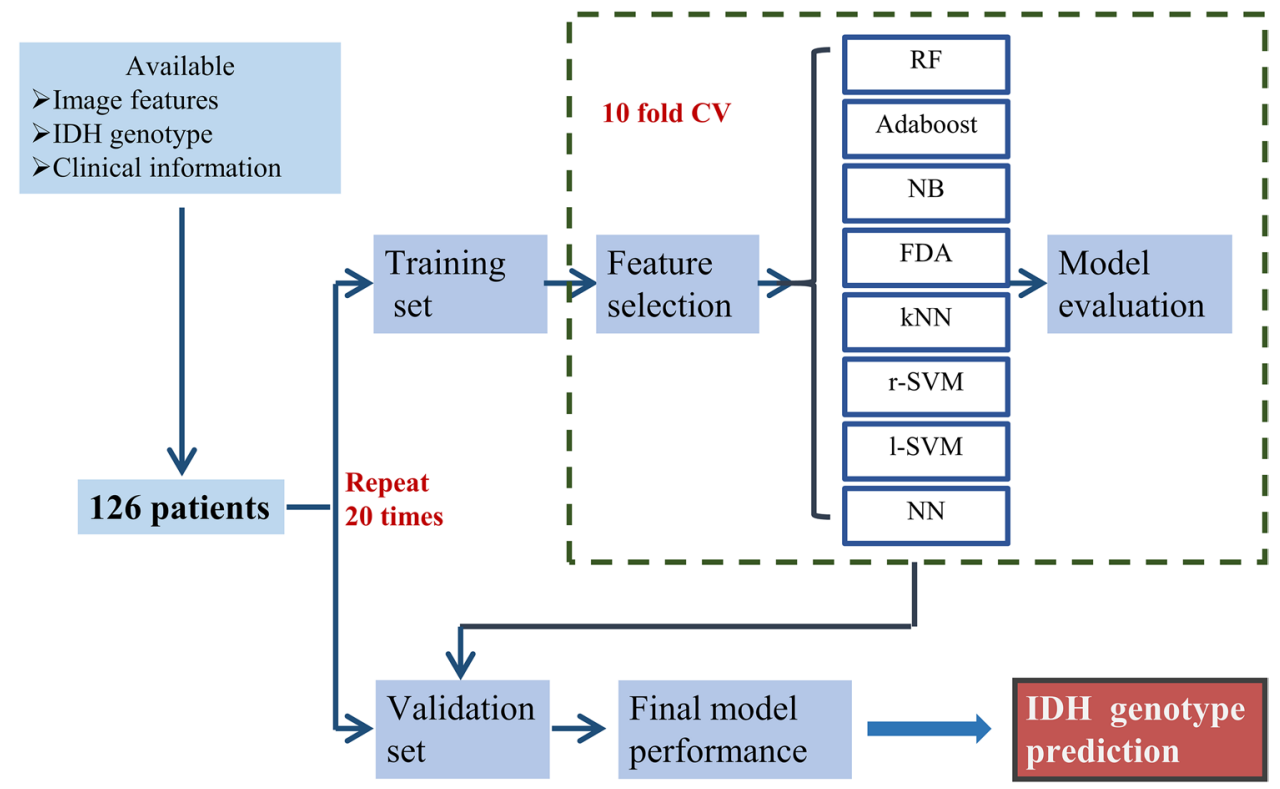

Table 1 Characteristics of 126 patients included in this study

\begin{tabular}{ll}
\hline & Total \\
\hline Patients & $n=126$ \\
Histology & \\
Grade II-III & $43(34.1 \%)$ \\
Grade IV & $83(65.9 \%)$ \\
IDH mutation & \\
WT & $87(69.0 \%)$ \\
Mutant & $39(31.0 \%)$ \\
Age at diagnosis (years) & \\
Mean \pm SD & $53.5 \pm 15.0$ \\
Range & $18-84$ \\
Gender & \\
Male & $67(53.2 \%)$ \\
Female & $59(46.8 \%)$ \\
\hline
\end{tabular}

network (NN). Short descriptions of the essential features of each method are presented in Table 2.

Patients were randomly assigned to either the training set or the validation set at a recommended ratio of 2:1 (Dobbin and Simon 2011). With over 700 radiomic features used for analyses, it was necessary to perform feature selection before classification model building, and minimum redundancy maximum relevance (MRMR) was applied to select the relevant and non-redundant features (De Jay et al. 2013; Ding and Peng 2005). Subsequently, the selected features were input as predictors for classification models constructing. Each model was trained using repeated (5 repeat iterations) tenfold cross-validation of the training set. The predictive performance was independently estimated in the validation set by quantifying the accuracy and area under the receiver operator characteristic (ROC) curve (AUC) (Robin et al. 2011). All the machine learning algorithms were conducted using the R caret package (Kuhn 2008). The parameters set for model training are listed in Supplementary Table 1.

We repeated the procedure of training and validation 20 times to achieve a robust estimate of the model performance. Different patients were assigned to the training and the validation set each time. The stability of each machine learning method was quantified through relative standard deviations (RSD) (Parmar et al. 2015). RSD\% is defined as:

$\mathrm{RSD} \%=\sigma \_\mathrm{AUC} / \mu \_\mathrm{AUC} \times 100 \%$,

where $\sigma \_$AUC is the standard deviation of the 20 AUC values and $\mu \_$AUC is the mean of the 20 AUC values. A lower RSD value corresponds to higher stability of the machine learning method.

\section{Classification models based on features of different subcategories}

To further explore the predictive values of different features in IDH genotype discrimination, the radiomic features were classified into six subcategories. The definitions of these features' subcategories are shown in Table 3. Feature selection was performed for each subcategory except spatial and TGM, as these two had few features. The predictive performance of the final model was independently estimated using the accuracy and AUC in the validation set. To achieve an average estimate of the model performance, we repeated the procedure 20 times.

All the analyses were completed using the R software (version 3.3.1). 
Table 2 The acronyms related to the used classification methods

\begin{tabular}{|c|c|c|}
\hline Acronym & Classification method name & Short descriptions \\
\hline RF & Random forest & $\begin{array}{l}\text { An ensemble learning method which grows substantial decision trees at training time, } \\
\text { then combines the individual decision of each tree to obtain the optimal classification } \\
\text { result (Liaw and Wiener 2002) }\end{array}$ \\
\hline Adaboost & Adaptive boosting & $\begin{array}{l}\text { A machine learning method which combines a multitude of relatively "weak classifiers" } \\
\text { to generate a "strong classifier" (Chen and Pan 2018) }\end{array}$ \\
\hline NB & Naive Bayes & A probabilistic classifier which is based on the Bayes' theorem (Rish 2001) \\
\hline FDA & Flexible discriminant analysis & $\begin{array}{l}\text { A flexible extension of linear discriminant analysis, in which a classification problem is } \\
\text { reduced to a regression problem (Hastie et al. 1994) }\end{array}$ \\
\hline $\mathrm{kNN}$ & k-Nearest neighbors & $\begin{array}{l}\text { A non-parametric learning algorithm assigning the new unknown example to the class } \\
\text { which the majority of its "k" nearest neighbors belong to (Bishop 2006; Duda et al. } \\
\text { 2000) }\end{array}$ \\
\hline r-SVM & $\begin{array}{l}\text { Support vector machines with radial basis } \\
\text { function kernel }\end{array}$ & $\begin{array}{l}\text { A classification algorithm of support vector machine using the radial basis function } \\
\text { kernel (Cristianini and Shawe-Taylor 2000) }\end{array}$ \\
\hline 1-SVM & Support vector machines with linear kernel & $\begin{array}{l}\text { A classification algorithm of support vector machine using the linear kernel (Cristianini } \\
\text { and Shawe-Taylor 2000) }\end{array}$ \\
\hline $\mathrm{NN}$ & Neural network & $\begin{array}{l}\text { A computing system which is inspired by how the nervous systems in a brain works } \\
\text { and contains a considerable amount of interconnected processing elements working in } \\
\text { unison for solving specific problems (Bishop 2006) }\end{array}$ \\
\hline
\end{tabular}

Table 3 Table defining the feature subcategory

\begin{tabular}{llr}
\hline Feature subcategory & Feature subcategory definition & Feature number \\
\hline Volume & Features of volumetric type & 21 \\
Spatial & Features of spatial type & 11 \\
TGM & Tumor growth model parameter & 6 \\
NET-hit & Histogram-based, intensity and textural features of the non-enhancing part of the tumor core & 222 \\
ET-hit & Histogram-based, intensity and textural features of the enhancing part of the tumor core & 222 \\
ED-hit & Histogram-based, intensity and textural features of the peritumoral edema & 222 \\
ALL & All features & 704 \\
\hline
\end{tabular}

\section{Results}

\section{Predictive performance of the classification methods}

In the current study, 126 patients (Table 1) were enrolled to investigate the effectiveness of eight machine learning methods (Table 2) for IDH genotype prediction. The workflow of classification model training and validation is displayed in Fig. 1. The accuracy and AUC were calculated to quantify the predictive performance of the classification methods. A total of 704 radiomic features were filtered by the feature selection method, MRMR. Then, the top 5, 10, 15, 20, 25, 30, 35 and 40 selected features were used to train the classifiers separately. The mean AUC and accuracy of all the classification methods trained with the selected features are reported in Supplementary Table 2 and Supplementary Table 3. In terms of accuracy, RF with top 20 selected features showed the highest predictive performance (accuracy $0.895 \pm 0.043$, range $0.825-0.975$ ), whereas $\mathrm{NN}$ with top 40 selected features displayed the lowest predictive performance (accuracy $0.829 \pm 0.064$, range 0.659-0.927) (Supplementary Table 2). As far as the AUC values were concerned, RF with top 15 selected features had the highest predictive performance (AUC $0.931 \pm 0.036$, range 0.866-1), while FDA with top 25 selected features showed the lowest predictive performance (AUC $0.875 \pm 0.057$, range 0.761-0.983) (Supplementary Table 3).

\section{Stability of the classification methods}

As the mean AUC value over all classifiers was highest when top 15 selected features were used (Fig. 2), the stability of classification methods with top 15 selected features was analyzed. The most stable classification model was $\mathrm{RF}(\mathrm{RSD}=3.87 \%)$, followed by Adaboost $(\mathrm{RSD}=4.76 \%)$, $\mathrm{NB}(\mathrm{RSD}=4.78 \%)$ and $\mathrm{r}-\mathrm{SVM}(\mathrm{RSD}=4.81 \%)$. FDA $(\mathrm{RSD}=5.82 \%)$ and $\mathrm{kNN}(\mathrm{RSD}=5.34 \%)$ had the lowest 


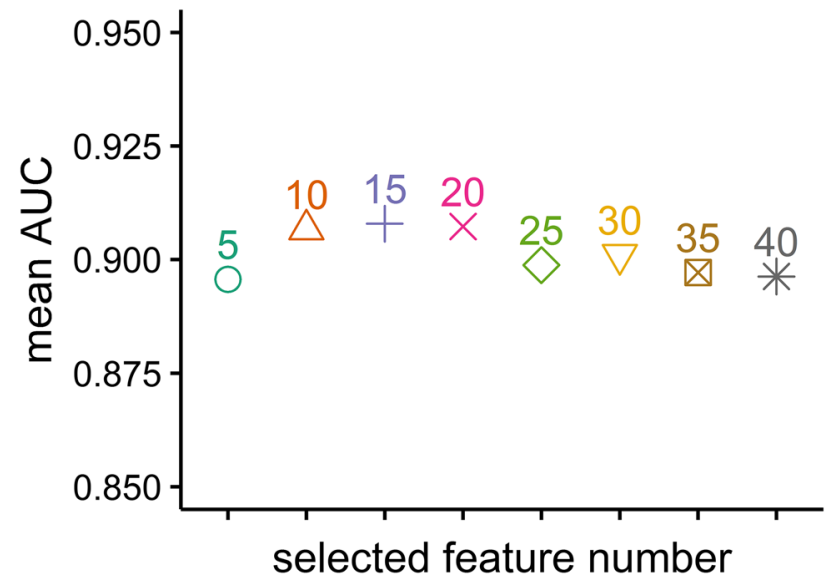

Fig. 2 Mean AUC values over all classifiers

stability among all the classification methods. Figure 3 shows an evaluation of model stability and predictive performance. We observed that RF $(\mathrm{RSD}=3.87 \%$, AUC 0.931 \pm 0.036 , accuracy $0.885 \pm 0.041$ ) out-performed other machine learning methods.

\section{Classification models based on features of different subcategories}

To investigate the value of the different features in IDH genotype predicting, the features were divided into six subcategories (Table 3). As RF had the highest stability and predictive performance among the eight classifiers, we used it to estimate the predictive role of different subcategories. As illustrated in Fig. 4, volume, NET-hit, ET-hit, and ED-hit out-performed spatial and TGM. The RF model based on volume features (AUC $0.928 \pm 0.035$, range $0.838-1$; accuracy $0.876 \pm 0.036$, range $0.805-0.927$ ) showed the highest predictive performance, whereas the RF model based on spatial features (AUC $0.566 \pm 0.052$, range $0.468-0.664$, accuracy $0.668 \pm 0.054$, range $0.585-0.756$ ) or TGM features (AUC $0.573 \pm 0.078$, range $0.424-0.743$, accuracy
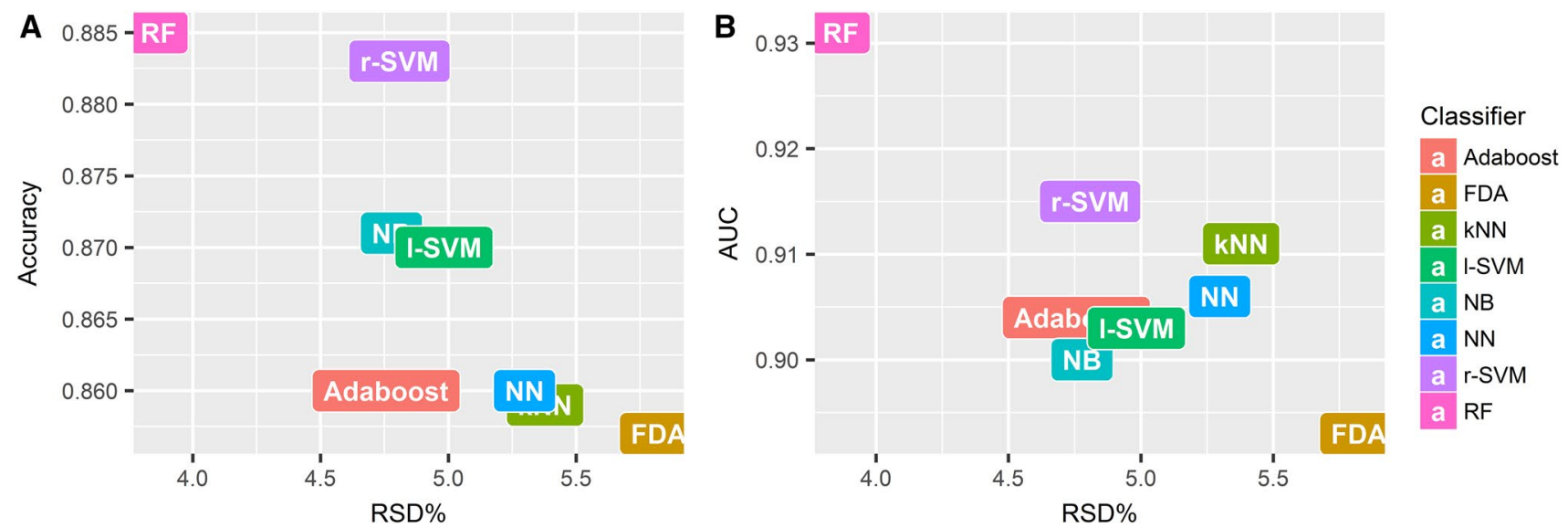

Fig. 3 Plots between model stability and predictive performance (Accuracy, AUC) of classifiers with top 15 selected features. a Accuracy vs RSD. b AUC vs RSD. RSD relative standard deviation; $A U C$ area under receiver operator characteristics curve

Fig. 4 Predictive performance of RF based on different feature subcategory. The top 15 selected features were used to train RF. a Accuracy; b AUC

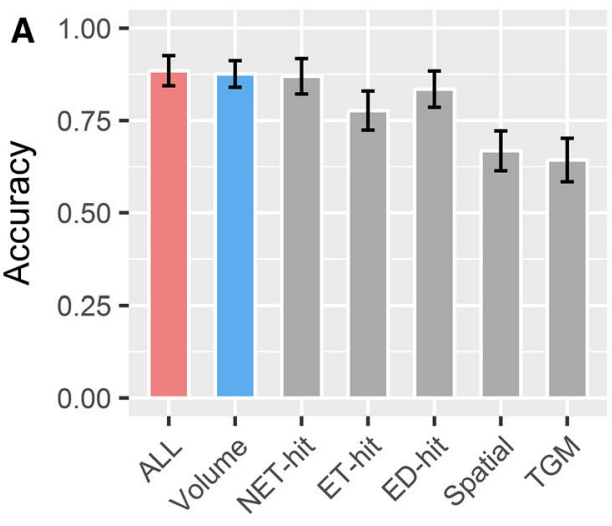

Feature category

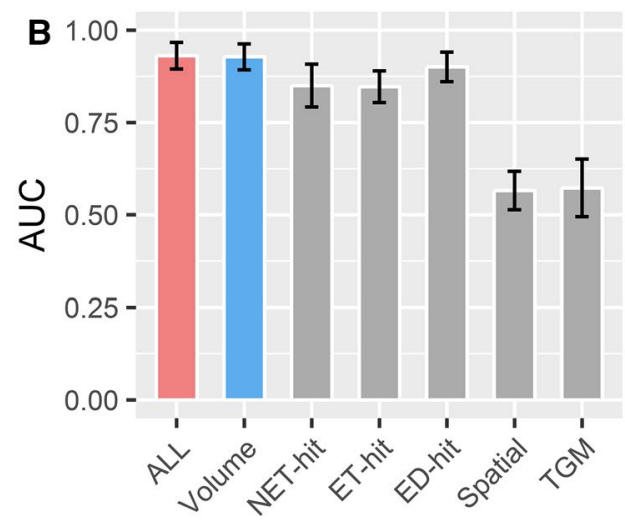

Feature category 
$0.643 \pm 0.059$, range $0.537-0.756$ ) had the lowest predictive performance.

\section{Discussion}

Precision oncology refers to customizing cancer care for individual patients. Such individual customization can maximize the benefits of prevention and treatment interventions while minimizing adverse effects. The success of precision oncology relies on accurately categorizing patients on the basis of their prognostic characteristics and responses to a specific treatment. As quantitative features extracted from medical images can be used to enhance the understanding of tumor characteristics, some studies have explored the value of radiomic features in precision oncology (Aerts et al. 2014; Nie et al. 2016; Rios Velazquez et al. 2017). Rios Velazquez et al. illustrated that radiomics-based machine learning model can be used to predict EGFR mutation status, which is an important biomarker for the treatment of lung cancer (Rios Velazquez et al. 2017). Nie et al. showed the potential of radiomics-based machine learning model to predict pathologic responses after pre-operative chemoradiation therapy for locally advanced rectal cancer (Nie et al. 2016). Results from these studies suggested that highly accurate and reliable classification models can promote the success of radiomics in precision oncology. Furthermore, identifying the optimal machine learning methods is recommended for different clinical tasks (Lambin et al. 2017).

Parmar et al. compared 12 machine learning methods in terms of their prognostic performance and stability for overall survival (OS) prediction in patients with lung cancer. They identified Random Forest (AUC: $0.66 \pm 0.03$ ) as the method with the highest prognostic performance and high stability (Parmar et al. 2015). Zhang et al. evaluated nine classification methods in terms of their predictive performance for the prediction of local failure and distant failure in advanced nasopharyngeal carcinoma. Random Forest (AUC $0.85 \pm 0.01$ ) and adaptive boosting (AUC $0.82 \pm 0.01$ ) were found to have the highest prognostic performance (Zhang et al. 2017). In another study, Parmar et al. investigated 11 machine learning methods in terms of their performance for predicting OS in patients with head and neck cancer. Bayesian (AUC 0.67, RSD: 11.28), Random Forest (AUC 0.61, RSD 7.36), and Nearest Neighbor (AUC 0.62, RSD 10.52) displayed high prognostic performance and stability (Parmar et al. 2015a, b). However, the optimal machine learning methods for IDH genotype predicting in patients with diffuse glioma have yet to be determined.

In the present study, we investigated and compared eight radiomics-based machine learning methods to preoperatively predict IDH genotype for diffuse gliomas. As described in the previous study, MRI images used for feature extraction were collected from eight institutes, which may make the model broadly applicable in the clinical practice (Bakas et al. 2017c). Moreover, the features analyzed in this study were extracted from labels segmented through a semiautomatic approach (Bakas et al. 2017c), which can reduce the variation of delineation between different observers and produce more reproducible and stable features (Parmar et al. 2014). More stable features may result in a more reliable classification model.

As over 700 quantitative radiomic features were analyzed in the current study, feature selection was performed. Feature selection is an exceedingly helpful strategy in data mining. It can help simplify the model, avoid the curse of dimensionality, and reduce over-fitting. MRMR was applied for feature selection in our analyses (Ding and Peng 2005). Finally, the top $5,10,15,20,25,30,35$ and 40 selected features were used to train the classifiers separately. The accuracy, AUC, and RSD were quantified to evaluate the predictive performance and stability of eight classical machine learning methods. The average performance over all classifiers was highest when the top 15 selected features were used, and RF (AUC $0.931 \pm 0.036$, accuracy $0.885 \pm 0.041$, RSD 3.867) had the highest predictive performance and stability. As one of the most frequently used machine learning algorithms in clinical classification problems (Parmar et al. 2015; Rios Velazquez et al. 2017; Zhang et al. 2017a, b), RF reduces over-fitting by bootstrap sampling and randomly selecting features at each split in the process of training (Liaw and Wiener 2002). The results from our analyses suggest that RF should be preferred with regard to predicting IDH genotype for patients with gliomas.

In the current study, we also evaluated and compared the predictive value of different feature subcategories. It has been shown that volume features were associated with molecular subtypes in glioma. Park et al. have shown that the IDH-mutant group had a smaller proportion of enhancing tumors in grade II and III gliomas (Park et al. 2017). Carrillo et al. demonstrated that the presence of non-contrast enhancing tumor was related to IDH mutation in grade IV gliomas (Carrillo et al. 2012). Textural features, which can quantify the intra-tumor heterogeneity by evaluating the gray-level intensity and position of the pixels within an image (Castellano et al. 2004; O'Connor et al. 2015), have also been applied to predict MGMT methylation status (Xi et al. 2017), EGFR expression (Li et al. 2018), and immune cell infiltration status (Narang et al. 2017) for gliomas. In our analysis, the feature subcategories volume, NET-hit, EThit, and ED-hit had high predictive performance (Fig. 4). Some of the patients with diffuse glioma did not demonstrate enhancement or edema on MRI scans. The models based on volume features or NET-hit features provide an opportunity to noninvasively and pre-operatively predict IDH genotype for these patients. 
This study has some limitations. First, larger sample sizes and external validation are required to assess the generalizability of our model. In the current study, we repeated the training procedure 20 times; each time different patients were assigned to the training and the validation set. Furthermore, the model predictive performance was repeatedly and independently evaluated in the validation set. These approaches enabled a proper estimation of our model generalizability. Second, recent studies have illustrated that diffusion-weighted imaging and magnetic resonance spectroscopy (MRS) have the potential to noninvasively identify IDH genotype for gliomas (Branzoli et al. 2017; Choi et al. 2016; Lee et al. 2015; Zhang et al. 2017a, b). The addition of imaging features from these modalities may improve the classification performance of our model. Third, the underlying biological mechanisms of how these features are correlated with IDH genotype in diffuse gliomas are presently unclear. Further research is needed to explore these mechanisms.

In summary, the role of MRI radiomic features in IDH genotype predicting and eight radiomics-based machine learning methods was compared and investigated in the present study. RF with top 15 selected features showed the highest predictive performance and stability (accuracy 0.885 \pm 0.041 , AUC $0.931 \pm 0.036$, RSD $3.87 \%$ ). These radiomics-based models maximized the value of the information contained in the medical images. Identification of an optimal radiomics-based machine learning method to noninvasively and pre-operatively predict IDH genotype can be valuable in the initial diagnostic evaluation and treatment planning for patients with diffuse glioma.

Funding This work was supported by the National Key R\&D Program of China (No. 2017YFC0107600) and the National Natural Science Foundation of China (No. 81773225).

\section{Compliance with ethical standards}

Conflict of interest The authors have no conflict of interest to declare.

Ethical approval All procedures performed in studies involving human participants were in accordance with the ethical standards of the institutional and/or national research committee and with the 1964 Helsinki Declaration and its later amendments or comparable ethical standards.

Informed consent All of the patients included in our study were de-identified by the Cancer Genome Atlas; hence, no Health Insurance Portability and Accountability Act or institutional review board approval was required and written informed consent was waived.

Open Access This article is distributed under the terms of the Creative Commons Attribution 4.0 International License (http://creativeco mmons.org/licenses/by/4.0/), which permits unrestricted use, distribution, and reproduction in any medium, provided you give appropriate credit to the original author(s) and the source, provide a link to the Creative Commons license, and indicate if changes were made.

\section{References}

Aerts HJ et al (2014) Decoding tumour phenotype by noninvasive imaging using a quantitative radiomics approach. Nat Commun 5:4006. https://doi.org/10.1038/ncomms5006

Bakas S, Akbari H, Sotiras A, Bilello M, Rozycki M, Kirby J, Freymann J, Farahani K, Davatzikos C (2017a) Segmentation labels and radiomic features for the pre-operative scans of the TCGALGG collection. Cancer Imaging Arch. https://doi.org/10.7937/ K9/TCIA.2017.GJQ7R0EF.

Bakas S, Akbari H, Sotiras A, Bilello M, Rozycki M, Kirby J, Freymann J, Farahani K, Davatzikos C (2017b) Segmentation labels and radiomic features for the pre-operative scans of the TCGAGBM collection. Cancer Imaging Arch. https://doi.org/10.7937/ K9/TCIA.2017.KLXWJJ1Q

Bakas $S$ et al (2017c) Advancing the cancer genome atlas glioma MRI collections with expert segmentation labels and radiomic features. Sci Data 4:170117. https://doi.org/10.1038/sdata .2017 .117

Bishop CM (2006) Pattern recognition and machine learning. Springer, New York

Branzoli F et al (2017) Highly specific determination of IDH status using edited in vivo magnetic resonance spectroscopy. Neuro Oncol. https://doi.org/10.1093/neuonc/nox214

Cancer Genome Atlas Research N et al (2015) Comprehensive, integrative genomic analysis of diffuse lower-grade gliomas. N Engl J Med 372:2481-2498. https://doi.org/10.1056/NEJMoa1402121

Carrillo JA et al (2012) Relationship between tumor enhancement, edema, IDH1 mutational status, MGMT promoter methylation, and survival in glioblastoma. AJNR 33:1349-1355. https://doi. org/10.3174/ajnr.A2950

Castellano G, Bonilha L, Li LM, Cendes F (2004) Texture analysis of medical images. Clin Radiol 59:1061-1069. https://doi. org/10.1016/j.crad.2004.07.008

Ceccarelli $\mathrm{M}$ et al (2016) Molecular profiling reveals biologically discrete subsets and pathways of progression in diffuse glioma. Cell 164:550-563. https://doi.org/10.1016/j.cell.2015.12.028

Chen P, Pan C (2018) Diabetes classification model based on boosting algorithms. BMC Bioinform 19:109. https://doi.org/10.1186/ s12859-018-2090-9

Choi C et al (2016) Prospective longitudinal analysis of 2-Hydroxyglutarate magnetic resonance spectroscopy identifies broad clinical utility for the management of patients with IDH-mutant glioma. J Clin Oncol 34:4030-4039. https://doi.org/10.1200/ jco.2016.67.1222

Clark K et al (2013) The cancer imaging archive (TCIA): maintaining and operating a public information repository. J Digit Imaging 26:1045-1057. https://doi.org/10.1007/s10278-013-9622-7

Cristianini N, Shawe-Taylor J (2000) An introduction to support vector machines and other kernel-based learning methods. Cambridge University Press, Cambridge

De Jay N, Papillon-Cavanagh S, Olsen C, El-Hachem N, Bontempi G, Haibe-Kains B (2013) mRMRe: an R package for parallelized mRMR ensemble feature selection. Bioinformatics 29:2365-2368. https://doi.org/10.1093/bioinformatics/btt383

Ding C, Peng H (2005) Minimum redundancy feature selection from microarray gene expression data. J Bioinform Comput Biol 3:185-205

Dobbin KK, Simon RM (2011) Optimally splitting cases for training and testing high dimensional classifiers. BMC Med Genom 4:31. https://doi.org/10.1186/1755-8794-4-31

Duda RO, Hart PE, Stork DG (2000) Pattern classification, 2nd edn. Wiley-Interscience, New York

Fehr D et al (2015) Automatic classification of prostate cancer Gleason scores from multiparametric magnetic resonance images. Proc 
Natl Acad Sci USA 112:E6265-E6273. https://doi.org/10.1073/ pnas. 1505935112

Hartmann C et al (2010) Patients with IDH1 wild type anaplastic astrocytomas exhibit worse prognosis than IDH1-mutated glioblastomas, and IDH1 mutation status accounts for the unfavorable prognostic effect of higher age: implications for classification of gliomas. Acta Neuropathol 120:707-718. https://doi.org/10.1007/ s00401-010-0781-z

Hastie T, Tibshirani R, Buja A (1994) Flexible discriminant analysis by optimal scoring. J Am Stat Assoc 89:1255-1270. https://doi. org/10.1080/01621459.1994.10476866

Huang Y et al (2016a) Radiomics signature: a potential biomarker for the prediction of disease-free survival in early-stage (I or II) non-small cell lung cancer. Radiology 281:947-957. https://doi. org/10.1148/radiol.2016152234

Huang YQ et al (2016b) Development and validation of a radiomics nomogram for preoperative prediction of lymph node metastasis in colorectal cancer. J Clin Oncol 34:2157-2164. https://doi. org/10.1200/JCO.2015.65.9128

Kuhn M (2008) Building predictive models in $\mathrm{R}$ using the caret package. J Stat Softw. https://doi.org/10.18637/jss.v028.i05

Lambin P et al (2012) Radiomics: extracting more information from medical images using advanced feature analysis. Eur J Cancer 48:441-446. https://doi.org/10.1016/j.ejca.2011.11.036

Lambin P et al (2017) Radiomics: the bridge between medical imaging and personalized medicine. Nat Rev Clin Oncol 14:749-762. https ://doi.org/10.1038/nrclinonc.2017.141

Lee $S$ et al (2015) Evaluation of the microenvironmental heterogeneity in high-grade gliomas with IDH1/2 gene mutation using histogram analysis of diffusion-weighted imaging and dynamic-susceptibility contrast perfusion imaging. J Neurooncol 121:141-150. https://doi.org/10.1007/s11060-014-1614-Z

Li H et al (2016) Quantitative MRI radiomics in the prediction of molecular classifications of breast cancer subtypes in the TCGA/ TCIA data set. NPJ Breast Cancer. https://doi.org/10.1038/npjbc ancer.2016.12

Li Y et al (2018) MRI features can predict EGFR expression in lower grade gliomas: A voxel-based radiomic analysis. Eur Radiol 28:356-362. https://doi.org/10.1007/s00330-017-4964-Z

Liaw A, Wiener M (2002) Classification and regression by randomForest. $\mathrm{R}$ news $2: 18-22$

Louis DN et al (2016) The 2016 World Health Organization classification of tumors of the central nervous system: a summary. Acta Neuropathol 131:803-820. https://doi.org/10.1007/s0040 1-016-1545-1

Macyszyn L et al (2016) Imaging patterns predict patient survival and molecular subtype in glioblastoma via machine learning techniques. Neuro Oncol 18:417-425. https://doi.org/10.1093/neuon c/nov 127

Narang S et al (2017) Tumor image-derived texture features are associated with CD3 T-cell infiltration status in glioblastoma. Oncotarget 8:101244-101254. https://doi.org/10.18632/oncotarget .20643

Nie K et al (2016) Rectal cancer: assessment of neoadjuvant chemoradiation outcome based on radiomics of multiparametric MRI. Clin Cancer Res 22:5256-5264. https://doi.org/10.1158/10780432.CCR-15-2997
O'Connor JP, Rose CJ, Waterton JC, Carano RA, Parker GJ, Jackson A (2015) Imaging intratumor heterogeneity: role in therapy response, resistance, and clinical outcome. Clin Cancer Res 21:249-257. https://doi.org/10.1158/1078-0432.CCR-14-0990

Ostrom QT, Gittleman H, Liao P, Vecchione-Koval T, Wolinsky Y, Kruchko C, Barnholtz-Sloan JS (2017) CBTRUS statistical report: primary brain and other central nervous system tumors diagnosed in the United States in 2010-2014. Neuro Oncol 19:v1-v88. https ://doi.org/10.1093/neuonc/nox 158

Park YW et al (2017) Prediction of IDH1-Mutation and 1p/19q-codeletion status using preoperative $\mathrm{mr}$ imaging phenotypes in lower grade gliomas. AJNR. https://doi.org/10.3174/ajnr.A5421

Parmar C et al (2014) Robust radiomics feature quantification using semiautomatic volumetric segmentation. PLoS One 9:e102107. https://doi.org/10.1371/journal.pone.0102107

Parmar C, Grossmann P, Rietveld D, Rietbergen MM, Lambin P, Aerts HJ (2015a) Radiomic machine-learning classifiers for prognostic biomarkers of head and neck cancer. Front Oncol 5:272. https:// doi.org/10.3389/fonc. 2015.00272

Parmar C, Grossmann P, Bussink J, Lambin P, Aerts HJ (2015b) Machine learning methods for quantitative radiomic biomarkers. Sci Rep 5:13087. https://doi.org/10.1038/srep13087

Parsons DW et al (2008) An integrated genomic analysis of human glioblastoma multiforme. Science 321:1807-1812. https://doi. org/10.1126/science.1164382

Rathore S, Akbari H, Rozycki M, Bakas S, Davatzikos C (2016) NIMG-20. Imaging pattern analysis reveals three distinct phenotypic subtypes of gbm with different survival rates. Neuro Oncol 18:vi128-vi128. https://doi.org/10.1093/neuonc/now212.532

Rios Velazquez E et al (2017) Somatic mutations drive distinct imaging phenotypes in lung cancer. Cancer Res 77:3922-3930. https://doi. org/10.1158/0008-5472.CAN-17-0122

Rish I (2001) An Empirical Study of the Naïve Bayes Classifier. In: IJCAI 2001 workshop on empirical methods in artificial intelligence, vol 3. IBM, New York, pp 41-46

Robin X, Turck N, Hainard A, Tiberti N, Lisacek F, Sanchez J-C, Müller M (2011) pROC: an open-source package for R and S + to analyze and compare ROC curves. BMC Bioinformatics 12:77. https://doi.org/10.1186/1471-2105-12-77

Xi YB et al (2017) Radiomics signature: a potential biomarker for the prediction of MGMT promoter methylation in glioblastoma. J Magn Reson Imaging. https://doi.org/10.1002/jmri.25860

Yan $\mathrm{H}$ et al (2009) IDH1 and IDH2 mutations in gliomas. N Engl J Med 360:765-773. https://doi.org/10.1056/NEJMoa0808710

$\mathrm{Yu}$ J et al (2017) Noninvasive IDH1 mutation estimation based on a quantitative radiomics approach for grade II glioma. Eur Radiol 27:3509-3522. https://doi.org/10.1007/s00330-016-4653-3

Zhang B et al (2017a) Multimodal MRI features predict isocitrate dehydrogenase genotype in high-grade gliomas. Neuro Oncol 19:109-117. https://doi.org/10.1093/neuonc/now121

Zhang B et al (2017b) Radiomic machine-learning classifiers for prognostic biomarkers of advanced nasopharyngeal carcinoma. Cancer Lett 403:21-27. https://doi.org/10.1016/j.canlet.2017.06.004

Zhang X et al (2018) Radiomics strategy for molecular subtype stratification of lower-grade glioma: detecting IDH and TP53 mutations based on multimodal MRI. J Magnetic Reson Imaging. https://doi. org/10.1002/jmri.25960 